\title{
Nonlinear Fluid Models for Biofluid Flow in Constricted Blood Vessels under Body Accelerations: A Comparative Study
}

\author{
D. S. Sankar ${ }^{1}$ and Atulya K. Nagar ${ }^{2}$ \\ ${ }^{1}$ School of Mathematical Sciences, University Science Malaysia, 11800 Penang, Malaysia \\ ${ }^{2}$ Centre for Applicable Mathematics and Systems Science, Department of Computer Science, \\ Liverpool Hope University, Hope Park, Liverpool L16 9JD, UK
}

Correspondence should be addressed to D. S. Sankar, sankar_ds@yahoo.co.in

Received 3 January 2012; Accepted 12 February 2012

Academic Editor: M. F. El-Amin

Copyright (C) 2012 D. S. Sankar and A. K. Nagar. This is an open access article distributed under the Creative Commons Attribution License, which permits unrestricted use, distribution, and reproduction in any medium, provided the original work is properly cited.

\begin{abstract}
Pulsatile flow of blood in constricted narrow arteries under periodic body acceleration is analyzed, modeling blood as non-Newtonian fluid models with yield stress such as (i) Herschel-Bulkley fluid model and (ii) Casson fluid model. The expressions for various flow quantities obtained by Sankar and Ismail (2010) for Herschel-Bulkley fluid model and Nagarani and Sarojamma (2008), in an improved form, for Casson fluid model are used to compute the data for comparing these fluid models. It is found that the plug core radius and wall shear stress are lower for H-B fluid model than those of the Casson fluid model. It is also noted that the plug flow velocity and flow rate are considerably higher for H-B fluid than those of the Casson fluid model. The estimates of the mean velocity and mean flow rate are considerably higher for H-B fluid model than those of the Casson fluid model.
\end{abstract}

\section{Introduction}

Atherosclerosis is an arterial disease in large and medium size blood vessels which involve in the complex interactions between the artery wall and blood flow and is caused by intravascular plaques leading to malfunctions of the cardiovascular system [1]. The intimal thickening of an artery is the initial process in the development of atherosclerosis and one of the most wide spread diseases in humans [2]. In atherosclerotic arteries, the lumen is typically narrowed and the wall is stiffened by the buildup of plaque with a lipid core and a fibromuscular cap, and the narrowing of lumen of the artery by the deposit of fats, lipids, cholesterol, and so forth is medically termed as stenosis formation [3]. Different shapes of 
stenoses are formed in arteries like axisymmetric, asymmetric, overlapping, and multiple and even sometimes it may be arbitrary in shape [4-7]. Once stenosis develops in an artery, its most serious consequences are the increased resistance and the associated reduction of blood flow to the vascular bed supplied by the artery $[8,9]$. Thus, the presence of a stenosis leads to the serious circulatory disorder. Hence, it is very useful to mathematically analyze the blood flow in stenosed arteries.

In many situations of our day to day life, we are exposed to body accelerations or vibrations, like swinging of kids in a cradle, vibration therapy applied to a patient with heart disease, travel of passengers in road vehicles, ships and flights, sudden movement of body in sports activities, and so forth [10,11]. Sometime, our whole body may be subjected to vibrations, like a passenger sitting in a bus/train, and so forth, while in some other occasions, specific part of our body might be subjected to vibrations, for example, in the operation of jack hammer or lathe machine, driver of a car, and so forth [12-14]. Prolonged exposure of our body to high level unintended external body accelerations causes serious health hazards due to the abnormal blood circulation [15-17]. Some of the symptoms which result from prolonged exposure of body acceleration are headache, abdominal pain, increase in pulse rate, venous pooling of blood in the extremities, loss of vision, hemorrhage in the face, neck, eye-sockets, lungs, and brain [18-20]. Thus, an adequate knowledge in this field is essential to the diagnosis and therapeutic treatment of some health problems, like vision loss, joint pain, and vascular disorder, and so forth, and also in the design of protective pads and machines. Hence, it is important to mathematically analyze and also to quantify the effects of periodic body accelerations in arteries of different diameters.

Due to the rheological importance of the body accelerations and the arterial stenosis, several theoretical studies were performed to understand their effects on the physiologically important flow quantities and also their consequences [15-20]. Blood shows anomalous viscous properties. Blood, when it flows through larger diameter arteries at high shear rates, it shows Newtonian character; whereas, when it flows in narrow diameter arteries at low shear rates, it exhibits remarkable non-Newtonian behavior [21, 22]. Many studies pertaining to blood flow analysis treated it as Newtonian fluid [4, 15, 23]. Several researchers used nonNewtonian fluids models for mathematical analysis of blood flow through narrow arteries with different shapes of stenosis under periodic body accelerations [24-27]. Casson and Herschel-Bulkley (H-B) fluid models are some of the non-Newtonian fluid models with yield stress and are widely used in the theoretical analysis of blood flow in narrow arteries $[28,29]$. The advantages of using H-B fluid model rather than Casson fluid model for modeling of blood flow in narrow arteries are mentioned below.

Chaturani and Samy [8] emphasized the use of H-B fluid model for blood flow modeling with the argument that when blood flows in arteries of diameter $0.095 \mathrm{~mm}$, it behaves like H-B fluid rather than other non-Newtonian fluids. Tu and Deville [21] pronounced that blood obeys Casson fluid's constitutive equation only at moderate shear rates, whereas H-B fluid model can be used still at low shear rates and represents fairly closely what is occurring in blood. Iida [30] reports "the velocity profiles of blood when it flows in the arterioles having diameter less than $0.1 \mathrm{~mm}$ are generally explained fairly by Casson and H-B fluid models. However, the velocity profiles of blood flow in the arterioles whose diameters are less than $0.065 \mathrm{~mm}$ do not conform to the Casson fluid model, but, can still be explained by H-B fluid model." Moreover, Casson fluid's constitutive equation has only one parameter, namely, the yield stress, whereas the H-B fluid's constitutive equation has one more parameter, namely, the power law index " $n$ " and 
thus one can obtain more detailed information about blood flow characteristics by using the H-B fluid model rather than Casson fluid model [31]. Hence, it is appropriate to treat blood as H-B fluid model rather than Casson fluid model when it flows through narrow arteries.

Sankar and Ismail [32] investigated the effects of periodic body accelerations in blood flow through narrow arteries with axisymmetric stenosis, treating blood as H-B fluid model. Nagarani and Sarojamma [33] mathematically analyzed the pulsatile flow of Casson fluid for blood flow through stenosed narrow arteries under body acceleration. The pulsatile flow of H-B fluid model and Casson fluid model for blood flow through narrow arteries with asymmetric stenosis under periodic body acceleration has not been studied so far, to the knowledge of the authors. Hence, in the present study, a comparative study is performed for the pulsatile flow H-B and Casson fluid models for blood flow in narrow arteries with asymmetric shapes of stenoses under periodic body acceleration. The expressions obtained in Sankar and Ismail [32] for shear stress, velocity distribution, wall shear stress, and flow rate are used to compute data for the present comparative study. The aforesaid flow quantities obtained by Nagarani and Sarojamma [33] for Casson fluid model in the corrected form are used in this study to compute data for performing the present comparative study. The layout of the paper is as follows.

Section 2 mathematically formulates the H-B and Casson fluid models for blood flow and applies the perturbation method of solution. In Section 3, the results of H-B fluid model and Casson fluid model for blood flow in axisymmetric and asymmetrically stenosed narrow arteries are compared. Some possible clinical applications to the present study are also given in Section 3. The main results are summarized in the concluding Section 4.

\section{Mathematical Formulation}

Consider an axially symmetric, laminar, pulsatile, and fully developed flow of blood (assumed to be incompressible) in the axial $(\bar{z})$ direction through a circular narrow artery with constriction. The constriction in the artery is assumed as due to the formation of stenosis in the lumen of the artery and is considered as mild. In this study, we consider the shape of the stenosis as asymmetric. The geometry of segment of a narrow artery with asymmetric shape of mild stenosis is shown in Figure 1(a). For different values of the stenosis shape parameter $m$, the asymmetric shapes of the stenoses are sketched in Figure 1(b). In Figure $1(b)$, one can notice the axisymmetric shape of stenosis when the stenosis shape parameter $m=2$. The segment of the artery under study is considered to be long enough so that the entrance, end, and special wall effects can be neglected. Due to the presence of the stenosis in the lumen of the segment of the artery, it is appropriate to treat the segment of the stenosed artery under study as rigid walled. Assume that there is periodical body acceleration in the region of blood flow and blood is modeled as non-Newtonian fluid model with yield stress. In this study, we use two different non-Newtonian fluid models with yield stress for blood flow simulations such as (i) Herschel-Bulkley (H-B) fluid and (ii) Casson fluid. Note that for particular values of the parameters, H-B fluid model's constitutive equation reduces to the constitutive equations of Newtonian fluid, power law fluid, and Bingham fluid. Also it is to be noted that Casson fluid model's constitutive equation reduces to the constitutive equation of Newtonian fluid when the yield stress parameter becomes zero. The cylindrical polar coordinate system $(\bar{r}, \bar{\psi}, \bar{z})$ has been used to analyze the blood flow. 


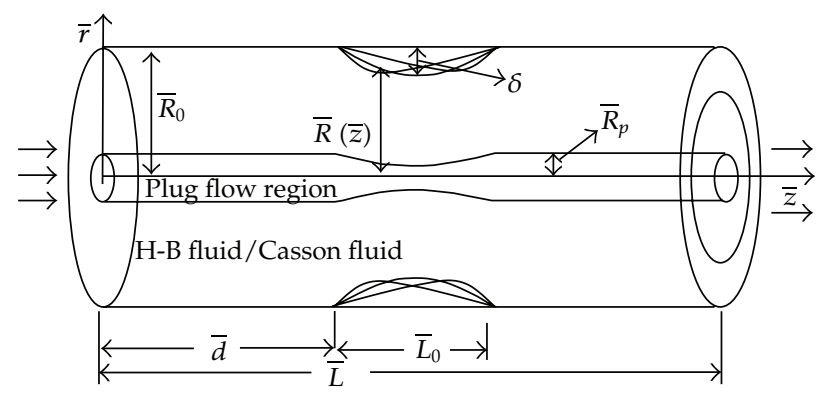

(a) Geometry of the stenosed artery in cylindrical polar coordinate system

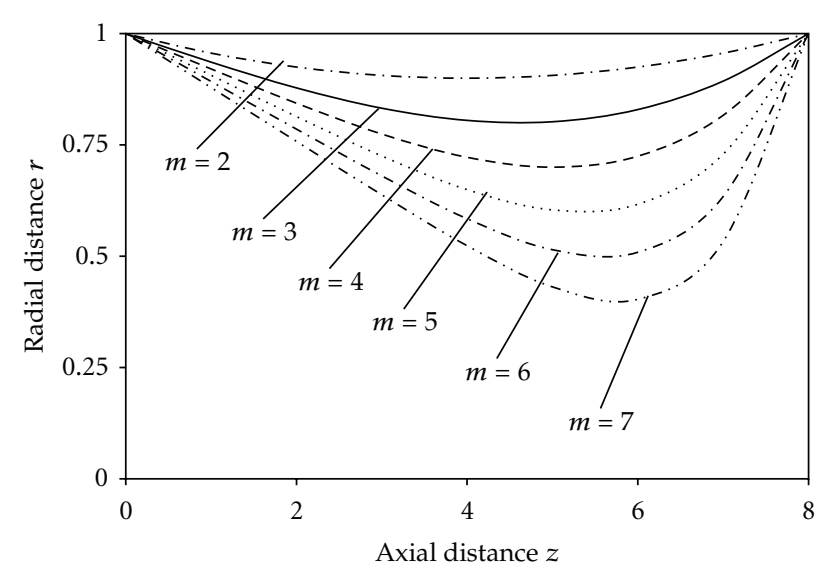

(b) Shapes of the arterial stenosis for different values of the stenosis shape parameter $m$

Figure 1: Pictorial description of segment of the artery with asymmetric stenosis.

\subsection{Herschel-Bulkley Fluid Model}

\subsubsection{Governing Equations and Boundary Conditions}

It has been reported that the radial velocity is negligibly small and can be neglected for a low Reynolds number flow in a narrow artery with mild stenosis. The momentum equations governing the blood flow in the axial and radial directions simplify, respectively, to [32]

$$
\begin{gathered}
\bar{\rho}_{H} \frac{\partial \bar{u}_{H}}{\partial \bar{t}}=-\frac{\partial \bar{p}}{\partial \bar{z}}-\frac{1}{\bar{r}} \frac{\partial}{\partial \bar{r}}\left(\bar{r}_{H}\right)+\bar{F}(\bar{t}), \\
0=\frac{\partial \bar{p}}{\partial \bar{r}}
\end{gathered}
$$


where $\bar{\rho}_{H}, \bar{u}_{H}$ are the density and axial component of the velocity of the H-B fluid, respectively; $\bar{p}$ is the pressure; $\bar{t}$ is the time; $\bar{\tau}_{H}=\left|\bar{\tau}_{\overline{r z}}\right|=-\bar{\tau}_{\overline{r z}}$ is the shear stress of the H$\mathrm{B}$ fluid; $\bar{F}(\bar{t})$ is the term which represents the effect of body acceleration and is given by

$$
\bar{F}(\bar{t})=\bar{a}_{0} \cos \left(\bar{\omega}_{b} \bar{t}+\phi\right)
$$

where $\bar{a}_{0}$ is the amplitude of the body acceleration, $\bar{\omega}_{b}=2 \pi \bar{f}_{b}, \bar{f}_{b}$ is the frequency in $\mathrm{Hz}$ and is assumed to be small so that the wave effect can be neglected [14], $\phi$ is the lead angle of $\bar{F}(\bar{t})$ with respect to the heart action. Since, the blood flow is assumed as pulsatile, it is appropriate to assume the pressure gradient as a periodic function as given below [25]:

$$
-\frac{\partial \bar{p}}{\partial \bar{z}}(\bar{z}, \bar{t})=\bar{A}_{0}+\bar{A}_{1} \cos \left(\bar{\omega}_{p} \bar{t}\right)
$$

where $\bar{A}_{0}$ is the steady component of the pressure gradient, $\bar{A}_{1}$ is the amplitude of the pulsatile component of the pressure gradient, and $\bar{\omega}_{p}=2 \pi \bar{f}_{p}, \bar{f}_{p}$ is the pulse frequency in $\mathrm{Hz}$ [23]. The constitutive equation of the H-B fluid (which represents blood) is given by

$$
\begin{gathered}
\bar{\tau}_{H}=\bar{\mu}_{H}^{1 / n}\left(\frac{-\partial \bar{u}_{H}}{\partial \bar{r}}\right)^{1 / n}+\bar{\tau}_{y} \quad \text { if } \tau_{H} \geq \tau_{y} \\
\frac{\partial \bar{u}_{H}}{\partial \bar{r}}=0 \quad \text { if } \bar{\tau}_{H} \leq \bar{\tau}_{y}
\end{gathered}
$$

where, $\bar{\tau}_{y}$ is the yield stress of the H-B fluid and $\bar{\mu}_{H}$ is the coefficient of viscosity of H-B fluid with dimension $\left(M L^{-1} T^{-2}\right)^{n} T$. The geometry of the asymmetric shape of stenosis in the arterial segment is mathematically represented by the following equation [34]:

$$
\frac{\bar{R}(\bar{z})}{\bar{R}_{0}}= \begin{cases}1-\bar{G}\left[\bar{L}_{0}^{m-1}(\bar{z}-\bar{d})-(\bar{z}-\bar{d})^{m}\right] & \text { if } \bar{d} \leq \bar{z} \leq \bar{d}+\bar{L}_{0} \\ 1 & \text { otherwise }\end{cases}
$$

where $\bar{G}=\left(\bar{\delta} / \bar{R}_{0} \bar{L}_{0}\right) m^{(m / m-1)} ; \bar{\delta}$ denotes the maximum height of the stenosis at $\bar{z}=\bar{d}+$ $\left(\bar{L}_{0} / m^{(m / m-1)}\right)$ such that $\bar{\delta} / \bar{R}_{0} \ll 1 ; \bar{L}_{0}$ is the length of the stenosis; $\bar{d}$ denotes its location; $\bar{R}(\bar{z})$ is the radius of the artery in the stenosed region; $\bar{R}_{0}$ is the radius of the normal artery. It is to be noted that (2.6) also represents the geometry of segment of the artery with axisymmetric stenosis when the stenosis shape parameter $m=2$. We make use of the following boundary conditions to solve the system of momentum and constitutive equations for the unknown velocity and shear stress:

$$
\begin{aligned}
& \bar{\tau}_{H} \text { is finite at } \bar{r}=0, \\
& \bar{u}_{H}=0 \quad \text { at } \bar{r}=\bar{R}(\bar{z}) .
\end{aligned}
$$




\subsubsection{Nondimensionalization}

Let us introduce the following nondimensional variables:

$$
\begin{gathered}
z=\frac{\bar{z}}{\bar{R}_{0}}, \quad R(z)=\frac{\bar{R}(\bar{z})}{\bar{R}_{0}}, \quad r=\frac{\bar{r}}{\bar{R}_{0}}, \quad t=\bar{t} \bar{\omega}, \quad \omega=\frac{\bar{\omega}_{b}}{\bar{\omega}_{p}}, \quad \delta=\frac{\bar{\delta}}{\bar{R}_{0}}, \quad u_{H}=\frac{\bar{u}_{H}}{\left(\bar{A}_{0} \bar{R}_{0}^{2} / 4 \bar{\mu}_{0}\right)}, \\
\tau_{H}=\frac{\bar{\tau}_{H}}{\left(\bar{A}_{0} \bar{R}_{0} / 2\right)}, \quad \theta=\frac{2 \bar{\tau}_{y}}{\bar{A}_{0} \bar{R}_{0}}, \quad \alpha_{H}^{2}=\frac{\bar{R}_{0}^{2} \bar{\omega} \bar{\rho}_{H}}{\bar{\mu}_{0}}, \quad e=\frac{\bar{A}_{1}}{\bar{A}_{0}}, \quad B=\frac{\bar{a}_{0}}{\bar{A}_{0}},
\end{gathered}
$$

where $\bar{\mu}_{0}=\bar{\mu}_{H}\left(2 / \bar{R}_{0} \bar{A}_{0}\right)^{n-1}$ having dimension as that of Newtonian fluid's viscosity $[22,34] ; \alpha_{H}$ is the generalized Wormersly frequency parameter or pulsatile Reynolds number, and when $n=1$, it reduces to the Newtonian fluid's pulsatile Reynolds number. Using nondimensional variables defined in (2.8), the momentum and constitutive equations (2.1) and (2.5) can be simplified to the following equations:

$$
\begin{gathered}
\alpha_{H}^{2} \frac{\partial u_{H}}{\partial t}=4(1+e \cos t)+4 B \cos (\omega t+\phi)-\frac{2}{r} \frac{\partial}{\partial r}\left(r \tau_{H}\right) \\
\tau_{H}=\left(-\frac{1}{2} \frac{\partial u_{H}}{\partial r}\right)^{1 / n}+\theta \quad \text { if } \tau_{H} \geq \theta \\
\frac{\partial u_{H}}{\partial r}=0 \quad \text { if } \tau_{H} \leq \theta
\end{gathered}
$$

The geometry of the asymmetric shape of the stenosis in the arterial segment in the nondimensional form reduces to the following equation:

$$
R(z)= \begin{cases}1-G\left[L_{0}^{m-1}(z-d)-(z-d)^{m}\right] & \text { if } d \leq z \leq d+L_{0} \\ 1 & \text { otherwise }\end{cases}
$$

where $G=\left(\bar{\delta} / \bar{R}_{0} \bar{L}_{0}\right) m^{(m / m-1)}$. The boundary conditions in the nondimensional form are

$$
\begin{gathered}
\tau_{H} \text { is finite at } r=0, \\
u_{H}=0 \quad \text { at } r=R .
\end{gathered}
$$

The volume flow rate in the nondimensional is given by

$$
Q(z, t)=4 \int_{0}^{R(z)} u_{H}(z, r, t) r d r
$$

where $Q(z, t)=\bar{Q}(\bar{z}, \bar{t}) /\left[\pi \bar{R}_{0}^{4} \bar{A}_{0} / 8 \bar{\mu}_{0}\right], \bar{Q}$ is the volumetric flow rate. 


\subsubsection{Perturbation Method of Solution}

Since, (2.9) and (2.10) form the system of nonlinear partial differential equations, it is not possible to get an exact solution to them. Thus, perturbation method is used to solve this system of nonlinear partial differential equations. Since, the present study deals with slow flow of blood (low Reynolds number flow) where the effect of pulsatile Reynolds number $\alpha_{H}$ is negligibly small and also it occurs naturally in the nondimensional form of the momentum equation, it is more appropriate to expand the unknowns $u_{H}$ and $\tau_{H}$ in (2.9) and (2.10) in the perturbation series about $\alpha_{H}^{2}$. Let us expand the velocity $u_{H}$ in the perturbation series about the square of the pulsatile Reynolds number $\alpha_{H}^{2}$ as below (where $\alpha_{H}^{2} \ll 1$ ):

$$
u_{H}(r, z, t)=u_{H 0}(r, z, t)+\alpha_{H}^{2} u_{H 1}(r, z, t)+\cdots
$$

Similarly, one can expand the shear stress $\tau_{H}(r, z, t)$, the plug core radius $R_{p}(z, t)$, the plug core velocity $u_{p}(z, t)$, and the plug core shear stress $\tau_{p}(z, t)$ in terms of $\alpha_{H}^{2}$. Substituting the perturbation series expansions of $u_{H}$ and $\tau_{H}$ in (2.9) and then equating the constant term and $\alpha_{H}^{2}$ term, we get

$$
\begin{gathered}
\frac{\partial}{\partial r}\left(r \tau_{H 0}\right)=2 r[(1+e \cos t)+B \cos (\omega t+\phi)], \\
\frac{\partial u_{H 0}}{\partial t}=-\frac{2}{r} \frac{\partial}{\partial r}\left(r \tau_{H 1}\right) .
\end{gathered}
$$

Using the binomial series approximation in (2.10) (assuming $\left.(\theta / \tau)^{2} \ll 1\right)$ and then applying the perturbation series expansions of $u_{H}$ and $\tau_{H}$ in the resulting equation and then equating the constant term and $\alpha_{H}^{2}$ term, one can obtain

$$
\begin{gathered}
-\frac{\partial u_{H 0}}{\partial r}=2 \tau_{H 0}^{n-1}\left[\tau_{H 0}-n \theta\right] \\
-\frac{\partial u_{H 1}}{\partial r}=2 n \tau_{H 0}^{n-2} \tau_{H 1}\left[\tau_{H 0}-(n-1) \theta\right] .
\end{gathered}
$$

Applying the perturbation series expansions of $u_{H}$ and $\tau_{H}$ in the boundary conditions (2.13), we obtain

$\tau_{H 0}, \tau_{H 1}$ are finite at $r=0$,

$$
u_{H 0}=0, u_{H 1}=0 \text { at } r=0 \text {. }
$$


Solving (2.16)-(2.17) with the help of the boundary conditions (2.18) for the unknowns $\tau_{P 0}, \tau_{P 1}, \tau_{H 0}, \tau_{H 1}, u_{P 0}, u_{P 1}, u_{H 0}$, and $u_{H 1}$, one can get the following expressions (detail of obtaining these expressions is given in [32]):

$$
\begin{aligned}
& \tau_{P 0}=g(t) R_{0 p}, \\
& \tau_{H 0}=g(t) r, \\
& u_{H 0}=2[g(t) R]^{n} R\left[\frac{1}{(n+1)}\left\{1-\left(\frac{r}{R}\right)^{n+1}\right\}-\left(\frac{q^{2}}{R}\right)\left\{1-\left(\frac{r}{R}\right)^{n}\right\}\right], \\
& u_{0 p}=2[g(t) R]^{n} R\left[\frac{1}{(n+1)}\left\{1-\left(\frac{q^{2}}{R}\right)^{n+1}\right\}-\left(\frac{q^{2}}{R}\right)\left\{1-\left(\frac{q^{2}}{R}\right)^{n}\right\}\right] \text {, } \\
& \tau_{P 1}=-[g(t) R]^{n} D R^{2}\left[\frac{n}{2(n+1)}\left(\frac{q^{2}}{R}\right)-\frac{(n-1)}{2}\left(\frac{q^{2}}{R}\right)^{2}-\frac{n}{2(n+1)}\left(\frac{q^{2}}{R}\right)^{n+2}\right], \\
& \tau_{H 1}=-[g(z) R]^{n} D R^{2}\left[\frac{n}{(n+1)(n+3)}\left\{\left(\frac{n+3}{2}\right)\left(\frac{r}{R}\right)-\left(\frac{r}{R}\right)^{n+2}\right\}\right. \\
& -\frac{(n-1)}{(n+2)}\left(\frac{q^{2}}{R}\right)\left\{\left(\frac{n+2}{2}\right)\left(\frac{r}{R}\right)-\left(\frac{r}{R}\right)^{n+1}\right\} \\
& \left.-\frac{3\left(n^{2}+2 n-2\right)}{2(n+2)(n+3)}\left(\frac{q^{2}}{R}\right)^{n+3}\left(\frac{R}{r}\right)\right] \\
& u_{H 1}=-2 n[g(t) R]^{2 n-1} D R^{3}\left[\frac{n}{2(n+1)^{2}(n+3)}\left\{(n+2)-(n+3)\left[\frac{r}{R}\right]^{n+1}+\left[\frac{r}{R}\right]^{2 n+2}\right\}\right. \\
& +\frac{(n-1)}{2(n+1)(n+2)(n+3)(2 n+1)}\left[\frac{q^{2}}{R}\right] \\
& \times\left\{(n+2)(n+3)(2 n+1)\left[\left(\frac{r}{R}\right)^{n}+\left(\frac{r}{R}\right)^{n+1}\right]\right. \\
& \left.-2\left[\left(2 n^{3}+9 n^{2}+11 n+3\right)+\left(2 n^{2}+6 n+3\right)\left[\frac{r}{R}\right]^{2 n+1}\right]\right\} \\
& +\frac{(n-1)^{2}}{2 n(n+2)}\left(\frac{q^{2}}{R}\right)^{2}\left\{(n+1)-(n+2)\left[\frac{r}{R}\right]^{n}+\left[\frac{r}{R}\right]^{2 n}\right\}
\end{aligned}
$$


Journal of Applied Mathematics

$$
\begin{aligned}
& +\frac{3\left(n^{2}+2 n-2\right)}{2(n-1)(n+2)(n+3)}\left(\frac{q^{2}}{R}\right)^{n+3}\left\{\left(\frac{r}{R}\right)^{n-1}-1\right\} \\
& \left.+\frac{3\left(n^{2}+2 n-2\right)(n-1)}{2(n-2)(n+2)(n+3)}\left(\frac{q^{2}}{R}\right)^{n+4}\left\{1-\left[\frac{r}{R}\right]^{n-2}\right\}\right], \\
u_{P 1}=-2 n[g(t) R]^{2 n-1} D R^{3}[ & \frac{n}{2(n+1)^{2}(n+3)}\left\{(n+2)-(n+3)\left[\frac{q^{2}}{R}\right]^{n+1}+\left[\frac{q^{2}}{R}\right]^{2 n+2}\right\} \\
& +\frac{(n-1)}{2(n+1)(n+2)(n+3)(2 n+1)}\left[\frac{q^{2}}{R}\right] \\
& \times\left\{(n+2)(n+3)(2 n+1)\left[\left(\frac{q^{2}}{R}\right)^{n}+\left(\frac{q^{2}}{R}\right)^{n+1}\right]\right. \\
& +\frac{(n-1)^{2}\left(\frac{q^{2}}{R}\right)^{2}\left\{(n+1)-(n+2)\left[\frac{q^{2}}{R}\right]^{n}+\left[\frac{q^{2}}{R}\right]^{2 n}\right\}}{2(n+2)} \\
& \left.\left.+\frac{3\left(n^{2}+2 n-2\right)(n-1)}{2(n-1)(n+2)(n+2)(n+3)}\left(\frac{q^{2}}{R}\right)^{n+4}\left\{1-\left[\frac{q^{2}}{R}\right]^{n-2}\right\}\right], \frac{q^{2}}{R}\right)^{n+3}\left\{\left(\frac{q^{2}}{R}\right)^{n-1}-1\right\}
\end{aligned}
$$

where $q^{2}=(\theta / g(t)),\left.r\right|_{\tau_{0 p}=\theta}=R_{0 p}=\theta / g(t)=q^{2}, g(t)=(1+e \cos t)+B \cos (\omega t+\phi)$, and $D=$ $(1 / g)(d g / d t)$. The wall shear stress $\tau_{w}$ is a physiologically important flow quantity which plays an important role in determining the aggregate sites of platelets [3]. The expression for wall shear stress $\tau_{w}$ is given by [32]

$$
\begin{aligned}
\tau_{w}= & \left(\tau_{H 0}+\alpha_{H}^{2} \tau_{H 1}\right)_{r=R} \\
= & {[g(t) R] } \\
& \times\left[1-\frac{(g(t) R)^{n-1} \alpha^{2} R^{2} B}{2(n+2)(n+3)}\right. \\
& \left.\quad \times\left\{n(n+2)-(n-1) n(n+3)\left(\frac{q^{2}}{R}\right)-3\left(n^{2}+2 n-2\right)\left(\frac{q^{2}}{R}\right)^{n+3}\right\}\right] .
\end{aligned}
$$


The expression for volumetric flow rate $Q(z, t)$ is obtained as below (see [32] for details):

$$
\begin{aligned}
Q(z, t)=4\left[\left(\int_{0}^{R_{0 p}} r u_{0 p} d r\right.\right. & \left.\left.+\int_{R_{0 p}}^{R} r u_{0} d r\right)+\alpha^{2}\left(\int_{0}^{R_{0 p}} r u_{1 p} d r+\int_{R_{0 p}}^{R} r u_{1} d r\right)\right] \\
=\frac{4[g(t) R]^{n} R^{3}}{(n+2)(n+3)}\left[\left\{(n+2)-n(n+3)\left(\frac{q^{2}}{R}\right)+\left(n^{2}+2 n-2\right)\left(\frac{q^{2}}{R}\right)^{n+3}\right\}\right. & \\
& -\alpha^{2}[g(t) R]^{n-1}\left(\frac{n D R^{2}}{4}\right) \\
\times & \left\{n-\frac{2 n(n-1)\left(4 n^{2}+12 n+5\right)}{(2 n+1)(2 n+3)}\left(\frac{q^{2}}{R}\right)\right. \\
& +\frac{n(n-1)^{2}(n+3)}{(n+1)}\left(\frac{q^{2}}{R}\right)^{2}+\frac{\left(n^{3}-2 n^{2}-11 n+6\right)}{(n+1)}\left(\frac{q^{2}}{R}\right)^{n+3} \\
& -\frac{(n-1)\left(n^{3}-2 n^{2}-11 n+6\right)}{n}\left(\frac{q^{2}}{R}\right)^{n+4} \\
& \left.\left.-\frac{\left(4 n^{5}+14 n^{4}-8 n^{3}-45 n^{2}-3 n+18\right)}{n(n+1)(2 n+3)}\left(\frac{q^{2}}{R}\right)^{2 n+4}\right\}\right] .
\end{aligned}
$$

The expression for the plug core radius is obtained as below [32]:

$$
R_{p}=q^{2}+\alpha^{2}[g(t) R]^{n-1}\left(\frac{n D R^{3}}{2(n+1)}\right)\left[\left(\frac{q^{2}}{R}\right)-\left(\frac{n^{2}-1}{n}\right)\left(\frac{q^{2}}{R}\right)^{2}-\left(\frac{q^{2}}{R}\right)^{n+2}\right] .
$$

The longitudinal impedance to flow in the artery is defined as

$$
\Lambda=\frac{P(t)}{Q(z, t)}
$$

where

$$
P(t)=4[1+e \cos (t)]
$$

is the pressure gradient in the nondimensional form. 


\subsection{Casson Fluid Model}

\subsubsection{Governing Equations and Boundary Conditions}

The momentum equations governing the blood flow in the axial and radial directions simplify, respectively, to [33]

$$
\begin{gathered}
\bar{\rho}_{C} \frac{\partial \bar{u}_{C}}{\partial \bar{t}}=-\frac{\partial \bar{p}}{\partial \bar{z}}-\frac{1}{\bar{r}} \frac{\partial}{\partial \bar{r}}\left(\overline{r \tau}_{C}\right)+\bar{F}(\bar{t}), \\
0=\frac{\partial \bar{p}}{\partial \bar{r}^{\prime}}
\end{gathered}
$$

where $\bar{u}_{C}$ and $\bar{\rho}_{C}$ are the axial component of the velocity and density of Casson fluid; $\bar{p}$ is the pressure; $\bar{t}$ is the time; $\bar{\tau}_{C}=\left|\bar{\tau}_{\overline{r z}}\right|=-\bar{\tau}_{\overline{r z}}$ is the shear stress of Casson fluid. Equations (2.3) and (2.4) which define mathematically the body acceleration term $\bar{F}(\bar{t})$ and pressure gradient $-(\partial \bar{p} / \partial \bar{z})$ are assumed in this subsection. Similarly, (2.6) which mathematically describes the geometry of the axisymmetric shape of stenosis and asymmetric shape of stenosis in the segment of the stenosed artery is also assumed in this subsection (the details of these assumptions can be found in Section 2.1.1) The constitutive equation of the Casson fluid model (which models blood) is defined as below:

$$
\begin{gathered}
\sqrt{\bar{\tau}_{C}}=\sqrt{\bar{\mu}_{C}\left(\frac{-\partial \bar{u}_{C}}{\partial \bar{r}}\right)}+\sqrt{\bar{\tau}_{y}} \quad \text { if } \tau_{C} \geq \tau_{y}, \\
\frac{\partial \bar{u}_{C}}{\partial \bar{r}}=0 \quad \text { if } \bar{\tau}_{C} \leq \bar{\tau}_{y}
\end{gathered}
$$

where $\bar{\tau}_{y}$ is the yield stress of Casson fluid and $\bar{\mu}_{C}$ is the coefficient of viscosity of Casson fluid with dimension $M L^{-1} T^{-1}$. The appropriate boundary conditions to solve the system of momentum and constitutive equations (2.25), (2.27), and (2.28) for the unknown velocity and shear stress are

$$
\begin{aligned}
& \bar{\tau}_{C} \text { is finite at } \bar{r}=0, \\
& \bar{u}_{C}=0 \quad \text { at } \bar{r}=\bar{R}(\bar{z}) .
\end{aligned}
$$




\subsubsection{Nondimensionalization}

Similar to (2.8), let us introduce the following nondimensional variables for the Casson fluid flow modeling as follows:

$$
\begin{gathered}
z=\frac{\bar{z}}{\bar{R}_{0}}, \quad R(z)=\frac{\bar{R}(\bar{z})}{\bar{R}_{0}}, \quad r=\frac{\bar{r}}{\bar{R}_{0}}, \quad t=\bar{t} \bar{\omega}, \quad \omega=\frac{\bar{\omega}_{b}}{\bar{\omega}_{p}}, \quad \delta=\frac{\bar{\delta}}{\bar{R}_{0}}, \quad u_{C}=\frac{\bar{u}_{C}}{\left(\bar{A}_{0} \bar{R}_{0}^{2} / 4 \bar{\mu}_{C}\right)}, \\
\tau_{C}=\frac{\bar{\tau}_{C}}{\left(\bar{A}_{0} \bar{R}_{0} / 2\right)}, \quad \theta=\frac{2 \bar{\tau}_{y}}{\bar{A}_{0} \bar{R}_{0}}, \quad \alpha_{C}^{2}=\frac{\bar{R}_{0}^{2} \overline{\omega \rho}_{C}}{\bar{\mu}_{C}}, \quad e=\frac{\bar{A}_{1}}{\bar{A}_{0}}, \quad B=\frac{\bar{a}_{0}}{\bar{A}_{0}},
\end{gathered}
$$

where $\alpha_{C}$ is the Wormersly frequency parameter or pulsatile Reynolds number of Casson fluid model. Use of the above nondimensional variables reduces the momentum and constitutive equations (2.25), (2.27), and (2.28), respectively, to the following equations:

$$
\begin{gathered}
\alpha_{C}^{2} \frac{\partial u_{C}}{\partial t}=4(1+e \cos t)+4 B \cos (\omega t+\phi)-\frac{2}{r} \frac{\partial}{\partial r}\left(r \tau_{C}\right), \\
\sqrt{\tau_{C}}=\sqrt{-\frac{1}{2} \frac{\partial u_{C}}{\partial r}}+\sqrt{\theta} \quad \text { if } \tau_{C} \geq \theta \\
\frac{\partial u_{C}}{\partial r}=0 \quad \text { if } \tau_{C} \leq \theta .
\end{gathered}
$$

Equation (2.12) which mathematically defines the nondimensional form of the geometry of the asymmetric shapes of stenosis in the arterial segment is assumed in this sub-section. The boundary conditions in the nondimensional form are

$$
\begin{gathered}
\tau_{C} \text { is finite at } r=0, \\
u_{C}=0 \quad \text { at } r=R .
\end{gathered}
$$

The volume flow rate in the nondimensional is given by

$$
Q=4 \int_{0}^{R(z)} u_{C}(z, r, t) r d r
$$

where $Q=\bar{Q} /\left[\pi \bar{R}_{0}^{4} \bar{A}_{0} / 8 \bar{\mu}_{C}\right], \bar{Q}$ is the volumetric flow rate. 


\subsubsection{Perturbation Method of Solution}

As described in Section 2.1.3, perturbation method is applied to solve the system of nonlinear partial differential equations (2.31) and (2.32). Let us expand the velocity $u_{C}$ in the perturbation series about the square of the pulsatile Reynolds number $\alpha_{C}^{2}$ as below (where $\alpha_{C}^{2} \ll 1$ ):

$$
u_{C}(r, z, t)=u_{C 0}(r, z, t)+\alpha_{C}^{2} u_{C 1}(r, z, t)+\cdots .
$$

Similarly, one can expand the shear stress $\tau_{C}(r, z, t)$, the plug core radius $R_{p}(z, t)$, the plug core velocity $u_{p}(z, t)$, and the plug core shear stress $\tau_{p}(z, t)$ in terms of $\alpha_{C}^{2}$. Substituting the perturbation series expansions of $u_{C}$ and $\tau_{C}$ in (2.31) and then equating the constant term and $\alpha_{C}^{2}$ term, one can obtain

$$
\begin{gathered}
\frac{\partial}{\partial r}\left(r \tau_{C 0}\right)=2 r[(1+e \cos t)+B \cos (\omega t+\phi)], \\
\frac{\partial u_{C 0}}{\partial t}=-\frac{2}{r} \frac{\partial}{\partial r}\left(r \tau_{C 1}\right) .
\end{gathered}
$$

Applying the perturbation series expansions of $u_{C}$ and $\tau_{C}$ in (2.32) and then equating the constant term and $\alpha_{C}^{2}$ term, we get

$$
\begin{gathered}
-\frac{\partial u_{C 0}}{\partial r}=2\left[\tau_{C 0}-2 \sqrt{\left(\frac{\theta}{\tau_{C 0}}\right)}+\theta\right], \\
-\frac{\partial u_{C 1}}{\partial r}=2 \tau_{C 1}\left[1-\sqrt{\left(\frac{\theta}{\tau_{C 0}}\right)}\right] .
\end{gathered}
$$

Applying the perturbation series expansions of $u_{C}$ and $\tau_{C}$ in the boundary conditions (2.34) and then equating the constant terms and $\alpha_{C}^{2}$ terms, one can get

$$
\begin{gathered}
\tau_{C 0}, \tau_{\mathrm{C} 1} \text { are finite at } r=0, \\
u_{\mathrm{C} 0}=0, \quad u_{C 1}=0 \quad \text { at } r=0 .
\end{gathered}
$$

Solving (2.37)-(2.38) with the help of the boundary conditions (2.39) for the unknowns $\tau_{P 0}, \tau_{P 1}, \tau_{C 0}, \tau_{C 1}, u_{P 0}, u_{P 1}, u_{C 0}$, and $u_{C 1}$, one can get the following expressions as in [33], but in a corrected form $((2.40)-(2.50))$ :

$$
\begin{gathered}
\tau_{P 0}=g(t) R_{0 p}, \\
\tau_{C 0}=g(t) r,
\end{gathered}
$$




$$
\begin{aligned}
& u_{C 0}=g(t) R^{2}\left[\left\{1-\left(\frac{r}{R}\right)^{2}\right\}-\frac{8}{3}\left(\frac{q}{\sqrt{R}}\right)\left\{1-\left(\frac{r}{R}\right)^{3 / 2}\right\}+\frac{2 q^{2}}{R}\left\{1-\left(\frac{r}{R}\right)\right\}\right], \\
& u_{P 0}=g(t) R^{2}\left[1-\frac{8}{3}\left(\frac{q}{\sqrt{R}}\right)+2\left(\frac{q^{2}}{R}\right)-\frac{1}{3}\left(\frac{q^{2}}{R}\right)^{2}\right] \\
& \tau_{P 1}=-\frac{g(t) D R^{5}}{12}\left(\frac{q^{2}}{R}\right)\left[3-4 \sqrt{\frac{q^{2}}{R}}+\left(\frac{q^{2}}{R}\right)^{2}\right] \\
& \tau_{C 1}=\frac{g(t) D R^{3}}{8} \\
& \times\left[2\left(\frac{r}{R}\right)-\left(\frac{r}{R}\right)^{3}-\left(\frac{q^{2}}{R}\right)^{4}\left(\frac{R}{r}\right)-\frac{8}{21} \sqrt{\frac{q^{2}}{R}}\left\{7\left(\frac{r}{R}\right)-4\left(\frac{r}{R}\right)^{5 / 2}-3\left(\frac{q^{2}}{R}\right)^{7 / 2}\left(\frac{R}{r}\right)\right\}\right], \\
& u_{C 1}=-g(t) D R^{4}\left[-\frac{1}{12}\left\{1-\left(\frac{r}{R}\right)^{2}\right\}-\frac{1}{3} \sqrt{\frac{q^{2}}{R}}\left\{1-\left(\frac{r}{R}\right)^{3 / 2}\right\}\right. \\
& -\frac{1}{16}\left\{1-\left(\frac{r}{R}\right)^{4}\right\}+\frac{53}{294} \sqrt{\frac{q^{2}}{R}}\left\{1-\left(\frac{r}{R}\right)^{7 / 2}\right\} \\
& +\frac{4}{9}\left(\frac{q^{2}}{R}\right)\left\{1-\left(\frac{r}{R}\right)^{3 / 2}\right\}-\frac{8}{63}\left(\frac{k^{2}}{R}\right)\left\{1-\left(\frac{r}{R}\right)^{3}\right\} \\
& \left.-\frac{1}{28}\left(\frac{q^{2}}{R}\right)^{4} \log \left(\frac{r}{R}\right)+\frac{1}{14}\left(\frac{q^{2}}{R}\right)^{9 / 2}\left\{1-\sqrt{\frac{R}{r}}\right\}\right] \text {, } \\
& u_{P 1}=-g(t) D R^{4}\left[-\frac{7}{48}+\frac{15}{98} \sqrt{\left(\frac{q^{2}}{R}\right)}-\frac{20}{63}\left(\frac{q^{2}}{R}\right)+\frac{5}{12}\left(\frac{q^{2}}{R}\right)^{2}\right. \\
& \left.-\frac{4}{9}\left(\frac{q^{2}}{R}\right)^{5 / 2}-\frac{439}{7056}\left(\frac{q^{2}}{R}\right)^{4}+\frac{1}{14}\left(\frac{q^{2}}{R}\right)^{9 / 2}-\frac{1}{28}\left(\frac{q^{2}}{R}\right)^{4} \log \left(\frac{q^{2}}{R}\right)\right] \text {, }
\end{aligned}
$$


where $q^{2}=(\theta / g(t)),\left.r\right|_{\tau_{0 p}=\theta}=R_{0 p}=\theta / g(t)=q^{2}, g(t)=(1+e \cos t)+B \cos (\omega t+\phi)$, and $D=(1 / g)(d g / d t)$. Using (2.41) and (2.45), the expression for wall shear stress $\tau_{w}$ is obtained as below:

$$
\tau_{w}=\left(\tau_{C 0}+\alpha_{C}^{2} \tau_{C 1}\right)_{r=R}=g(t) R\left[1-\frac{\alpha_{C}^{2} R^{2} D}{8}\left\{1-\frac{8}{7}\left(\frac{q}{\sqrt{R}}\right)+\frac{1}{7}\left(\frac{q^{2}}{R}\right)^{4}\right\}\right] .
$$

The expression for volumetric flow rate $Q(z, t)$ is obtained as below:

$$
\begin{aligned}
& Q(z, t)=4\left[\left(\int_{0}^{R_{0 P}} r u_{0 p} d r+\int_{R_{0 P}}^{R} r u_{0} d r\right)+\alpha^{2}\left(\int_{0}^{R_{0 P}} r u_{1 p} d r+\int_{R_{0 P}}^{R} r u_{1} d r\right)\right] \\
&=g(t) R^{4}\left[1-\frac{16}{7}\left(\frac{q}{\sqrt{R}}\right)+\frac{4}{3}\left(\frac{q^{2}}{R}\right)-\frac{1}{21}\left(\frac{q^{2}}{R}\right)^{4}-\alpha_{C}^{2} R^{6} g(t) D\right. \\
& \times\left\{\frac{1}{6}-\frac{30}{77}\left(\frac{q}{\sqrt{R}}\right)+\frac{8}{35}\left(\frac{q^{2}}{R}\right)-\frac{1}{3}\left(\frac{q^{2}}{R}\right)^{5 / 2}+\frac{1}{14}\left(\frac{q^{2}}{R}\right)^{9 / 2}\right. \\
&\left.\left.\quad-\frac{41}{770}\left(\frac{q^{2}}{R}\right)^{6}-\frac{1}{14}\left(\frac{q^{2}}{R}\right)^{6} \log \left(\frac{q^{2}}{R}\right)+\frac{1}{14}\left(\frac{q^{2}}{R}\right)^{4}\left(1-\left(\frac{q^{2}}{R}\right)^{2}\right) \log (q)\right\}\right] .
\end{aligned}
$$

The expression for the plug core radius is obtained as below [33]:

$$
R_{p}=q^{2}-\frac{D \alpha_{C}^{2} R^{3}}{4}\left[\left(\frac{q^{2}}{R}\right)-\frac{4}{3}\left(\frac{q^{2}}{R}\right)^{3 / 2}+\frac{1}{3}\left(\frac{q^{2}}{R}\right)^{3}\right]
$$

The longitudinal impedance to flow in the artery is defined as

$$
\Lambda=\frac{P(t)}{Q(z, t)} .
$$

\section{Numerical Simulation of the Results}

The main objective of the present mathematical analysis is to compare the H-B and Casson fluid models for blood flow in constricted arteries and spell out the advantageous of using H-B fluid model rather than Casson fluid for the mathematical modeling of blood flow in a narrow artery with asymmetric stenosis. It is also aimed to bring out the effect of body acceleration, stenosis shape parameter, yield stress, and pressure gradient on the physiologically important flow quantities such as plug core radius, plug flow velocity, velocity distribution, flow rate, wall shear stress, and longitudinal impedance to flow. The different parameters used in this analysis and their range of values are given below [32-35]. 


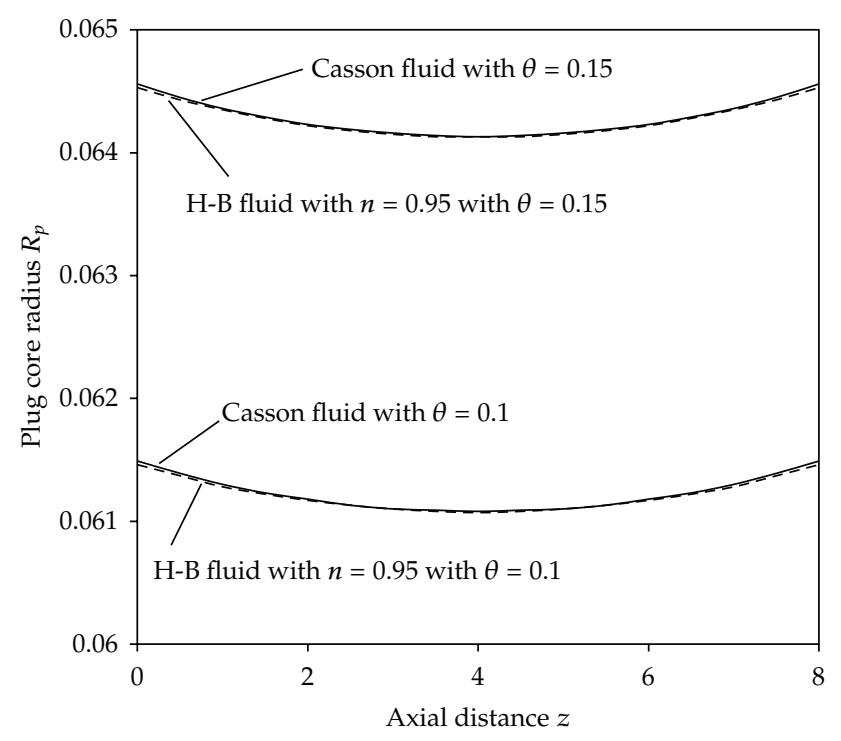

Figure 2: Variation of plug core radius with axial distance for $\mathrm{H}-\mathrm{B}$ and Casson fluid models with different values of yield stress $\theta$ and with $\delta=0.15, \alpha_{H}=\alpha_{C}=0.2, B=2, e=\phi=0.7$, and $t=45^{\circ}$.

Yield stress $\theta$ : 0-0.3; power law index $n$ : 0.95-1.05; pressure gradient $e$ : $0-1$; body acceleration B: 0-2; frequency parameter $\omega: 0-1$; pulsatile Reynolds numbers $\alpha_{H}$ and $\alpha_{C}: 0.2-$ 0.7 ; lead angle $\phi: 0.2-0.5$; asymmetry parameter $m: 2-7$; stenosis depth $\delta: 0-0.2$.

\subsection{Plug Core Radius}

The variation of the plug core with axial distance in axisymmetric stenosed artery $(m=2)$ for different values of the yield stress of H-B and Casson fluid models with $\delta=0.15, B=2, \alpha_{H}=$ $\alpha_{C}=0.2, e=\phi=0.7$ and $t=45^{\circ}$ is shown in Figure 2 . It is observed that the plug core radius decreases slowly when the axial variable $z$ increases from 0 to 4 and then it increases when $z$ increases further from 4 to 8 . The plug core radius is minimum at the centre of the stenosis $(z=4)$, since the stenosis is axisymmetric. The plug core radius of the H-B fluid model is slightly lower than that of the Casson fluid model. One can note that the plug core radius increases very significantly when the yield stress of the flowing blood increases. Figure 3 sketches the variation of plug core radius with pressure gradient ratio in asymmetrically stenosed artery $(m=4)$ for H-B and Casson fluid models and for different values of the body acceleration parameter with $\theta=\delta=0.1, t=60^{\circ}, \phi=0.7, m=4$, and $z=4$. It is noticed that the plug core radius decreases rapidly with the increase of the pressure gradient ratio $e$ from 0 to 0.5 and then it decreases slowly with the increase of the pressure gradient ratio $e$ from 0.5 to 1 . It is seen that plug core radius increases significantly with the increase of the body acceleration parameter $B$. Figures 2 and 3 bring out the influence of the non-Newtonian behavior of blood and the effects of body acceleration and pressure gradient on the plug core radius when blood flows in asymmetrically stenosed artery. 


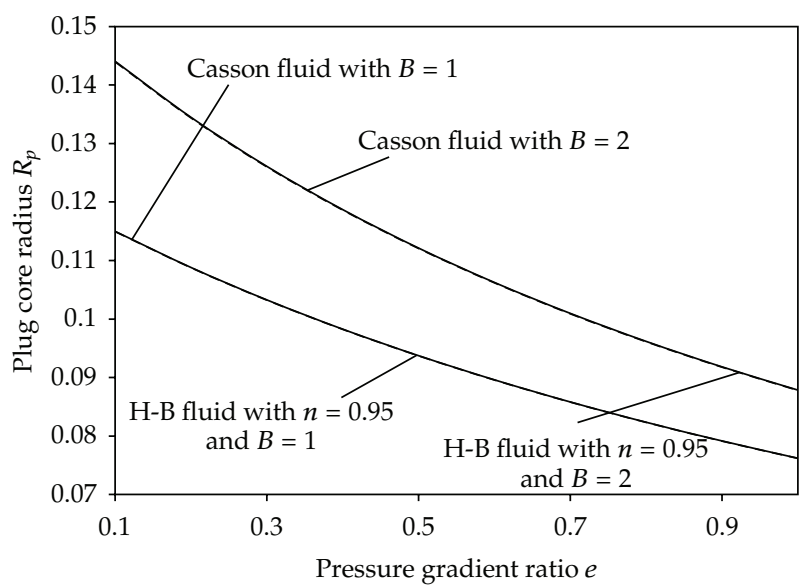

Figure 3: Variation of plug core radius with pressure gradient for $\mathrm{H}-\mathrm{B}$ and Casson fluids and for different values of body acceleration parameter $B$ with $\theta=\delta=0.1, t=60^{\circ}, \phi=0.7, m=4$, and $z=4$.

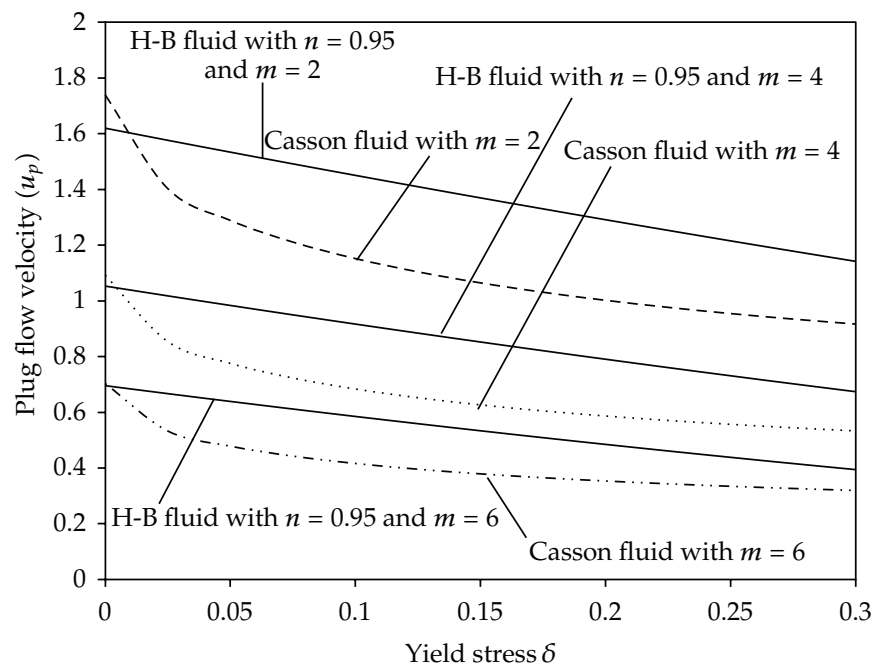

Figure 4: Variation of plug flow velocity with yield stress for $\mathrm{H}-\mathrm{B}$ and Casson fluid models and for different values of stenosis shape parameter $m$ with $e=0.5, \phi=0.2, t=60^{\circ}, z=4, \omega=0.5, B=1$, and $\delta=0.1$.

\subsection{Plug Flow Velocity}

Figure 4 shows the variation of the plug flow velocity with yield stress for H-B and Casson fluid models and for different values of the stenosis shape parameter with $e=0.5, \phi=0.2$, $z=4, t=60^{\circ}, \omega=0.5, B=1$, and $\delta=0.1$. It is noted that for H-B fluid model, the plug flow velocity decreases very slowly with the increase of the yield stress, whereas, in the case of Casson fluid model, it decreases rapidly when the yield stress $\theta$ increases from 0 to 0.05 and then it decreases slowly with the increase of the yield stress from 0.05 to 0.3 . It is seen that the plug flow velocity is considerably higher for H-B fluid model than that of the Casson fluid model. One can easily observe that the plug flow velocity decreases significantly with the increase of the stenosis shape parameter $m$. The variation of plug flow velocity with axial 


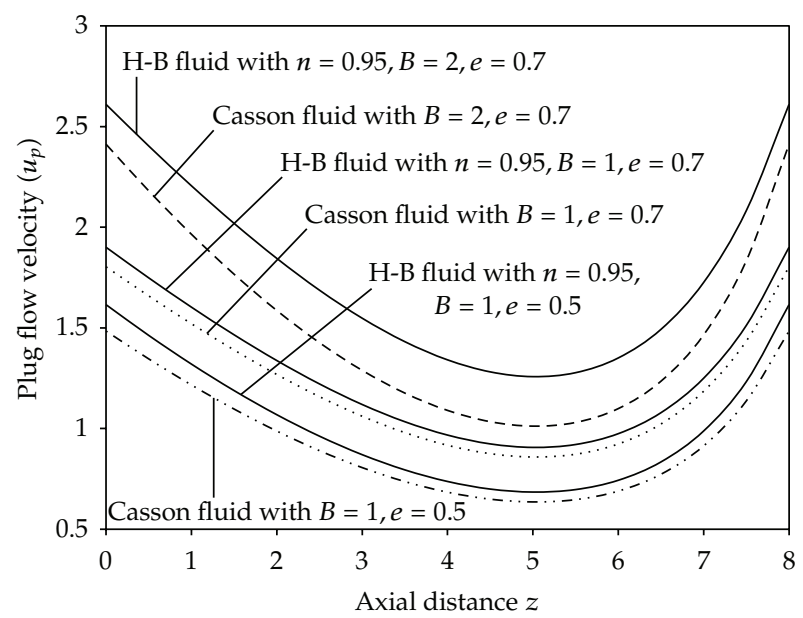

Figure 5: Variation of plug flow velocity with axial distance for H-B and Casson fluids and for different values of $B$ and $e$ with $\delta=\theta=0.1, m=4, t=60^{\circ}, \phi=0.2$, and $\omega=0.5$.

distance for $\mathrm{H}-\mathrm{B}$ and Casson fluid models and for different values of the body acceleration $B$ and pressure gradient ratio $e$ with $\delta=\theta=0.1, m=4, t=60^{\circ}, \phi=0.2$, and $\omega=0.5$ is depicted in Figure 5. It is seen that the plug flow velocity skews more to the right-hand side in the axial direction which is attributed by the skewness of the stenosis. It is clear that the plug flow velocity increases considerably with the increase of the body acceleration parameter $B$ and pressure gradient ratio $e$. Figures 4 and 5 show the non-Newtonian character of blood and effects of body acceleration, pressure gradient, and asymmetry of the stenosis on the plug flow velocity of blood when it flows through a constricted artery.

\subsection{Velocity Distribution}

Figure 6 sketches the velocity distribution for H-B and Casson fluid models and for different values of yield stress $\theta$, stenosis depth $\delta$ with $m=2, e=0.2, \alpha_{H}=\alpha_{C}=0.5, \phi=0.2, \omega=1$, $t=60^{\circ}$, and $B=1$. It is observed that the velocity of $\mathrm{H}-\mathrm{B}$ fluid model is considerably higher than that of Casson fluid model. It is also found that the velocity of the blood flow decreases with the increase of the yield stress $\theta$ and stenosis depth $\delta$. But the decrease in the velocity is considerable when the stenosis depth $\delta$ increases, whereas it decreases significantly with the increase of the yield stress. It is of interest to note that the velocity distribution of $\mathrm{H}-\mathrm{B}$ fluid with $\delta=0.2$ and $\theta=0.05$ and $B=0$ is in good agreement with the corresponding plot in Figure 6 of Sankar and Lee [34]. It is also to be noted that the velocity distribution of Casson fluid with $\delta=0.2, \theta=0.01$, and $B=0$ is in good agreement with the corresponding plot in Figure 6 of Siddiqui et al. [35].

\subsection{Flow Rate}

The variation of flow rate with pressure gradient ratio for $\mathrm{H}-\mathrm{B}$ and Casson fluid models and for different values of the power law index $n$, body acceleration parameter $B$, and stenosis shape parameter $m$ with $\theta=\delta=0.1, \alpha_{H}=\alpha_{C}=\phi=0.2, z=4, t=60^{\circ}$, and $\omega=1$ is shown 


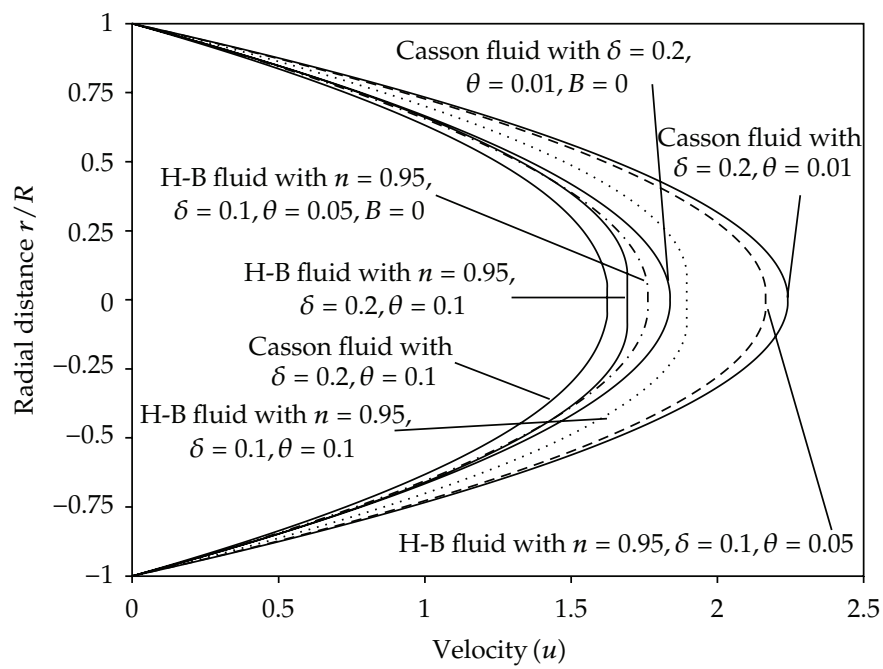

Figure 6: Velocity distribution for different fluid models with $e=0.2, \alpha_{H}=\alpha_{C}=0.5, \phi=0.2, \omega=1, t=60^{\circ}$, and $B=1$.

in Figure 7. It is seen that the flow rate increases with the pressure gradient ratio $e$. But the increase in the flow rate is linear for H-B fluid model and almost constant for Casson fluid model. For a given set of values of the parameters, the flow rate for H-B fluid model is considerably higher than that of the Casson fluid model. It is also clear that for a given set of values of $n$ and $m$, the flow rate increases considerably with the increase of the body acceleration parameter $B$. One can observe that for fixed values of $n$ and $B$, the flow rate decreases significantly with the increase of the stenosis shape parameter $m$. When the power law index $n$ increases from 0.95 to 1.05 and all the other parameters were held constant, the flow rate decreases slightly when the range of the pressure gradient ratio $e$ is $0-0.5$ and this behavior is reversed when the range of the pressure gradient ratio $e$ is 0.5 to 1 . Figure 7 brings out the effects of body acceleration and stenosis shape on the flow rate of blood when it flows through narrow artery with mild stenosis.

\subsection{Wall Shear Stress}

Figure 8 shows the variation of wall shear stress with frequency ratio for H-B and Casson fluid models and for different values of the $\phi$ (lead angle), $\alpha_{H}$ (pulsatile Reynolds number for H-B fluid model), and $\alpha_{C}$ (pulsatile Reynolds number of Casson fluid model) with $m=$ $2, \theta=\delta=0.1, e=0.5, B=1, z=4$, and $t=60^{\circ}$. It is seen that the wall shear stress decreases slightly nonlinearly with frequency ratio for lower values of the pulsatile Reynolds numbers $\alpha_{H}$ and $\alpha_{C}$ and lead angle $\phi$, and it decreases linearly with frequency ratio for higher values of the pulsatile Reynolds numbers $\alpha_{H}$ and $\alpha_{C}$ and lead angle $\phi$. It is found that for a given set of values of the parameters, the wall shear stress is marginally lower for H-B fluid model than that of the Casson fluid model. Also, one can note that for fixed value of the lead angle $\phi$, the wall shear stress decreases significantly with the increase of the pulsatile Reynolds numbers $\alpha_{H}$ and $\alpha_{C}$. It is also observed that the wall shear stress decreases marginally with the increase of the lead angle $\phi$ when all the other parameters were kept as invariables. Figure 8 spells out 


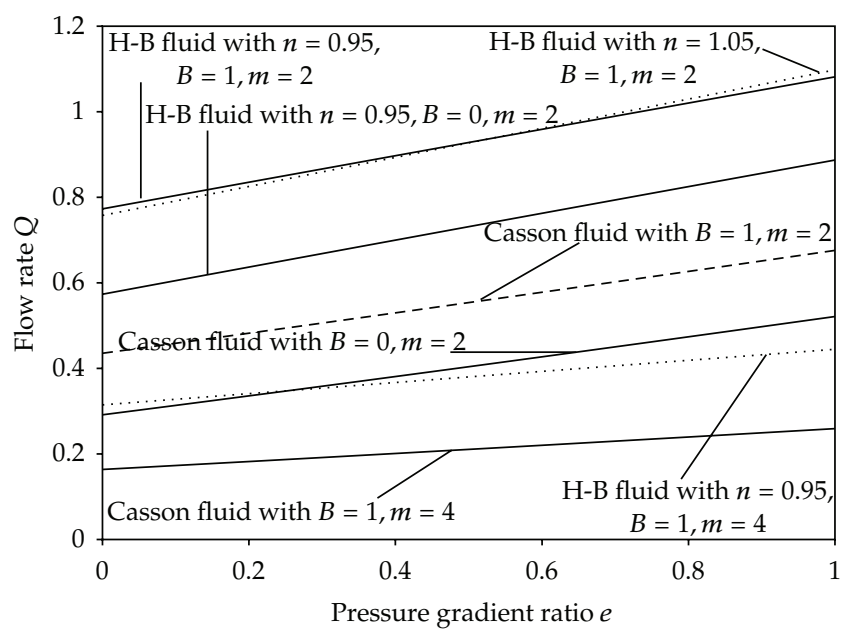

Figure 7: Variation of flow rate with pressure gradient for H-B and Casson fluid models and for different values of $B$ and $m$ with $\theta=\delta=0.1, \alpha_{H}=\alpha_{C}=\phi=0.2, z=4, t=60^{\circ}$, and $\omega=1$.

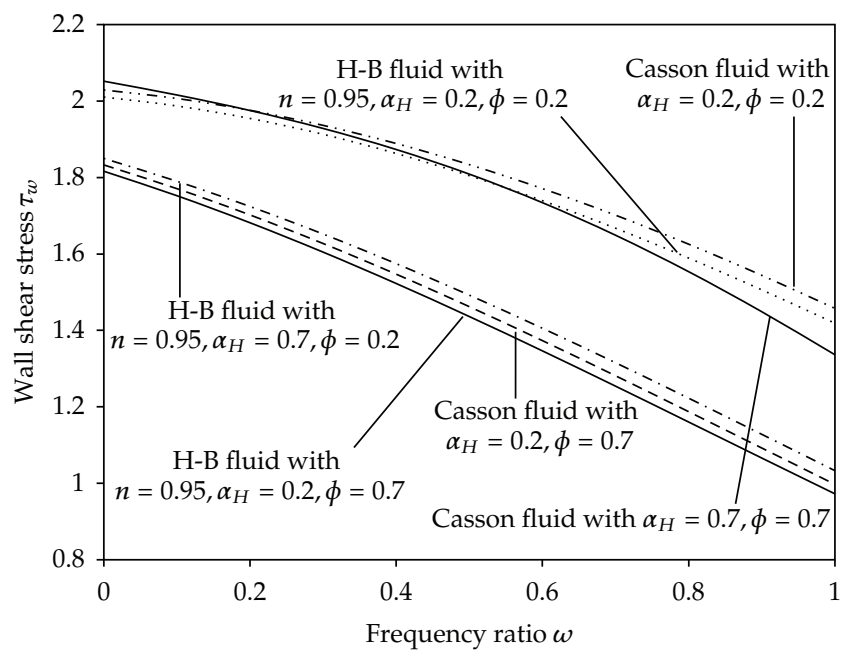

Figure 8: Variation of wall shear stress with frequency ratio for H-B and Casson fluids and for different values of $\alpha_{H}, \alpha_{C}$ and $\phi$ with $\theta=\delta=0.1, m=2, e=0.5, B=1, z=4$, and $t=60^{\circ}$.

the effects of pulsatility and non-Newtonian character of blood on the wall shear stress when it flows in a narrow artery with mild stenosis.

\subsection{Longitudinal Impedance to Flow}

The variation of the longitudinal impedance to flow with axial distance for different values of the stenosis shape parameter $m$ and body acceleration parameter $B$ with $\theta=\delta=0.1, t=60^{\circ}$, $\alpha_{H}=\alpha_{C}=\phi=0.2, e=0.5$, and $\omega=1$ is depicted in Figures 9(a) (for H-B fluid model) and 9(b) (Casson fluid model). It is noticed that the longitudinal impedance to flow increases with the increase of the axial variable $z$ from 0 to the point where the stenosis depth is maximum 


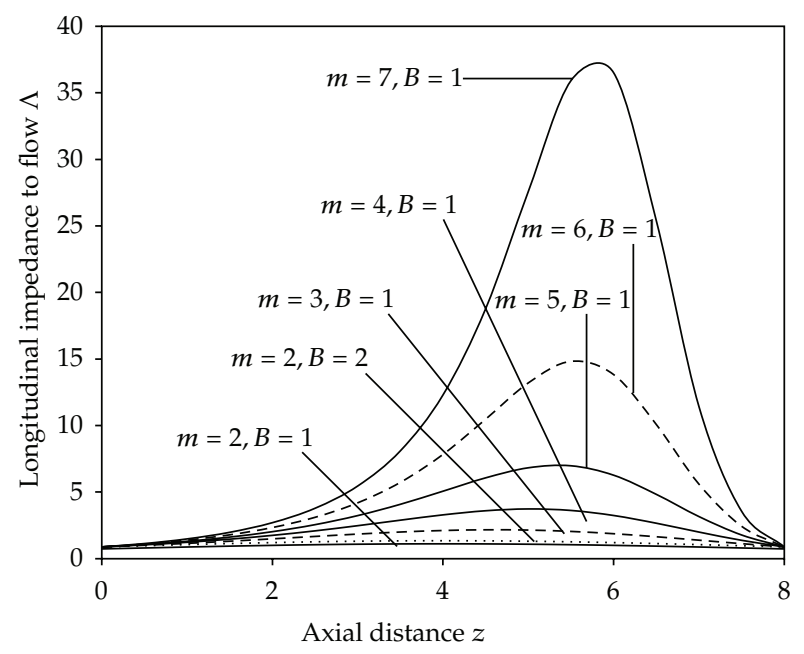

(a) Herschel-Bulkley fluid with $n=0.95$

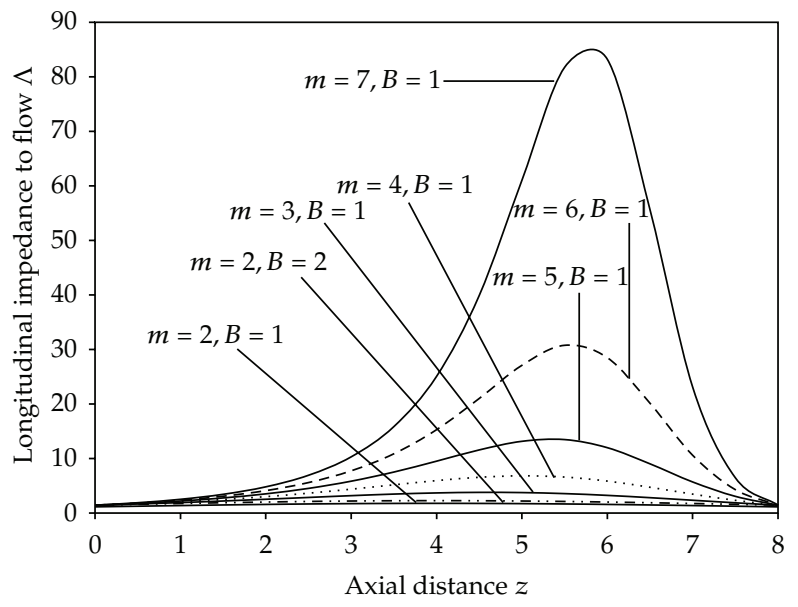

(b) Casson fluid

Figure 9: Variation of longitudinal impedance to flow with axial distance for H-B and Casson fluid models and for different values of $m$ and $B$ with $\theta=\delta=0.1, t=60^{\circ}, \alpha_{H}=\alpha_{C}=\phi=0.2, e=0.5$, and $\omega=1$.

and then it decreases as the axial variable $z$ increases further from that point to 8 . One can see the significant increase in the longitudinal impedance to flow when the stenosis shape parameter $m$ increases and marginal increase in the longitudinal impedance to flow when the body acceleration parameter $B$ increases. It is also clear that for the same set of values of the parameters, the longitudinal impedance to flow is significantly lower for H-B fluid model than that of the Casson fluid model. Figures 9(a) and 9(b) bring out the effects of body acceleration and asymmetry of the stenosis shape on the longitudinal impedance to blood flow.

The increase in the longitudinal impedance to blood flow due to the asymmetry shape of the stenosis is defined as the ratio between the longitudinal impedance to flow of a fluid model for a given set of values of the parameters in an artery with asymmetric stenosis and the longitudinal impedance of the same fluid model and for the same set of values 
Table 1: Estimates of the increase in the longitudinal impedance to flow due to the increase in the stenosis shape parameter and body acceleration with $\delta=\theta=0.1, e=0.5, \omega=1, \alpha_{H}=\alpha_{C}=\phi=0.2, z=4$, and $t=60^{\circ}$.

\begin{tabular}{ccccccc}
\hline$m$ & \multicolumn{3}{c}{ H-B fluid model } & \multicolumn{3}{c}{ Casson fluid model } \\
& $B=0$ & $B=1$ & $B=2$ & $B=0$ & $B=1$ & $B=2$ \\
\hline 3 & 1.5759 & 1.5698 & 1.5657 & 1.6401 & 1.6282 & 1.6202 \\
4 & 2.4616 & 2.4419 & 2.4289 & 2.6690 & 2.6289 & 2.6022 \\
5 & 3.8118 & 3.7642 & 3.7331 & 4.3105 & 4.2098 & 4.1433 \\
6 & 5.9191 & 5.8153 & 5.7484 & 6.9964 & 6.7688 & 6.6201 \\
7 & 9.3531 & 9.1338 & 8.9944 & 11.6016 & 11.1022 & 10.7803 \\
\hline
\end{tabular}

Table 2: Physiological data for different arteries.

\begin{tabular}{lcccc}
\hline S. no. & Artery & Radius $\left(\times 10^{-2} \mathrm{~m}\right)$ & $A_{0}\left(\times 10 \mathrm{Kg} \mathrm{m}^{-2} \mathrm{~s}^{-1}\right)$ & $A_{1}\left(\times 10 \mathrm{Kg} \mathrm{m}^{-2} \mathrm{~s}^{-1}\right)$ \\
\hline 1 & Aorta & 1.0 & 7.3 & 1.46 \\
2 & Femoral & 0.5 & 32.0 & 6.4 \\
3 & Carotid & 0.4 & 50.0 & 10.0 \\
4 & Coronary & 0.15 & 698.65 & 139.74 \\
5 & Arteriole & 0.008 & 2000.0 & 400 \\
\hline
\end{tabular}

of the parameters in that artery with axisymmetric stenosis. The estimates of the increase in the longitudinal impedance to flow are computed in Table 1 for different values of the stenosis shape parameter $m$ and body acceleration parameter $B$ with $\delta=\theta=0.1, e=0.5$, $\omega=1, z=4, \alpha_{H}=\alpha_{C}=\phi=0.2$, and $t=60^{\circ}$. It is observed that the estimates of the increase in the longitudinal impedance to flow increase considerably when the stenosis shape parameter $m$ increases and they decrease slightly when the body acceleration parameter $B$ increases. Hence, the longitudinal impedance to flow is significantly higher in the arteries with asymmetric shape of the stenosis compared to that in the arteries with axisymmetric stenosis. It is also noted that the presence of the body acceleration decreases the longitudinal impedance to blood flow considerably.

\subsection{Some Possible Clinical Applications}

To discuss some possible clinical applications of the present study, the data (for different types of arteries, their corresponding radii, steady and pulsatile pressure gradient values) reported by Chaturani and Wassf Issac [23] are given in Table 2 and are used in this applications part of our study. For these clinical data (given in Table 2), the estimates of the mean velocity of H-B and Casson fluid models for different values of the stenosis shape parameter $m$ and different values of the body acceleration parameter $B$ with $\theta=\delta=0.1, t=60^{\circ}, \omega=1, z=4, \phi=0.2, \alpha_{H}$ $=\alpha_{C}=0.2$, and $e=0.2$ are computed in Table 3 . It is recorded that the estimates of the mean velocity increase significantly with the increase of the artery radius, except in arterioles. It is also found that the estimates of the mean velocity of H-B fluid model are marginally higher than those of the Casson fluid model. It is noted that the mean velocity increases considerably with the increase of the body acceleration parameter $B$ and the reverse behavior is found when the stenosis shape parameter $m$ increases.

For the clinical data given in Table 2, the estimates of the mean flow rate of H-B and Casson fluid models are computed in Table 4 for different values of the stenosis shape parameter $m$ and different values of the body acceleration parameter $B$ with $\theta=\delta=0.1, \omega$ 
Table 3: Estimates of mean velocity of H-B and Casson fluid models for different values of $m$ and $B$ in arteries with different radii with $\theta=\delta=0.1, t=60^{\circ}, \omega=1, z=4, e=0.2$, and $\alpha_{H}=\alpha_{C}=\phi=0.2$.

\begin{tabular}{lccccccccc}
\hline & & \multicolumn{3}{c}{ H-B fluid model $\left(\times 10^{-2} \mathrm{~m} \mathrm{~s}^{-1}\right)$} & \multicolumn{3}{c}{ Casson fluid model $\left(\times 10^{-2} \mathrm{~m} \mathrm{~s}^{-1}\right)$} \\
S. no. & Artery type & \multicolumn{2}{c}{$m=2$} & \multicolumn{2}{c}{$m=4$} & \multicolumn{2}{c}{$m=2$} & \multicolumn{2}{c}{$m=4$} \\
& & $B=0$ & $B=1$ & $B=0$ & $B=1$ & $B=0$ & $B=1$ & $B=0$ & $B=1$ \\
\hline 1 & Aorta & 40.28 & 46.25 & 36.35 & 41.82 & 37.46 & 42.89 & 33.18 & 37.88 \\
2 & Femoral & 46.78 & 52.38 & 40.22 & 45.41 & 42.55 & 49.67 & 37.69 & 42.47 \\
3 & Carotid & 46.78 & 52.38 & 40.22 & 45.41 & 42.55 & 49.67 & 37.69 & 42.47 \\
4 & Coronary & 93.85 & 97.55 & 89.45 & 93.48 & 89.24 & 94.38 & 86.12 & 90.59 \\
5 & Arteriole & 0.62 & 0.75 & 0.55 & 0.68 & 0.57 & 0.69 & 0.51 & 0.63 \\
\hline
\end{tabular}

Table 4: Estimates of mean flow rate of H-B and Casson fluid models for different values of $m$ and $B$ in arteries with different radii with $\theta=\delta=0.1, t=60^{\circ}, \omega=1, z=4, e=0.2$, and $\alpha_{H}=\alpha_{C}=\phi=0.2$.

\begin{tabular}{lccccccccc}
\hline & & \multicolumn{3}{c}{ H-B fluid model $\left(\times 10^{-2} \mathrm{~m} \mathrm{~s}^{-1}\right)$} & \multicolumn{3}{c}{ Casson fluid model $\left(\times 10^{-2} \mathrm{~m} \mathrm{~s}^{-1}\right)$} \\
S. no. Artery type & \multicolumn{2}{c}{$m=2$} & \multicolumn{2}{c}{$m=4$} & \multicolumn{2}{c}{$m=2$} & \multicolumn{2}{c}{$m=4$} \\
& & $B=0$ & $B=1$ & $B=0$ & $B=1$ & $B=0$ & $B=1$ & $B=0$ & $B=1$ \\
\hline 1 & Aorta & 65.43 & 72.54 & 61.42 & 68.72 & 61.49 & 66.58 & 55.24 & 62.66 \\
2 & Femoral & 14.87 & 20.38 & 12.66 & 17.41 & 11.57 & 16.93 & 10.41 & 15.28 \\
3 & Carotid & 9.56 & 13.15 & 8.43 & 11.85 & 7.94 & 11.27 & 6.25 & 9.49 \\
4 & Coronary & 3.12 & 3.55 & 2.85 & 3.25 & 2.75 & 3.15 & 2.37 & 2.84 \\
5 & Arteriole & $70.8 E-6$ & $82.5 E-6$ & $67.3 E-6$ & $74.6 E-6$ & $65.8 E-6$ & 75.61 & $61.8 E-6$ & $69.7 E-6$ \\
\hline
\end{tabular}

$=1, t=60^{\circ}, z=4, \phi=0.2, \alpha_{H}=\alpha_{C}=0.2$, and $e=0.2$. It is observed that the estimates of the mean flow rate decrease very significantly with the increase of the artery radius. It is also found that the estimates of the mean flow rate of H-B fluid model are considerably higher than those of the Casson fluid model. It is noted that the estimates of the mean flow rate increase significantly with the increase of the body acceleration parameter $B$ and the reverse behavior is recorded when the stenosis shape parameter $m$ increases.

\section{Conclusions}

The present mathematical analysis brings out various interesting rheological properties of blood when it flows through narrow stenosed arteries with body acceleration, treating it as different non-Newtonian fluid models with yield stress such as (i) Herschel-Bulkley fluid model and (ii) Casson fluid model. By the use of appropriate mathematical expression for the geometry of segment of the stenosed artery, both axisymmetric and asymmetric shapes of stenoses are considered to study the effects of stenosis shape and size on the physiologically important quantities. Some major findings of this mathematical analysis are summarized below.

(i) The plug core radius, wall shear stress, and longitudinal impedance to flow are marginally lower for H-B fluid model than those of the Casson fluid model.

(ii) The plug flow velocity, velocity distribution, and flow rate are considerably higher for H-B fluid model than those of the Casson fluid model. 
(iii) The plug core radius and longitudinal impedance to flow increase significantly with the increase of the stenosis shape parameter, and the reverse behavior is observed for plug flow velocity, velocity distribution, and flow rate.

(iv) The estimates of the mean velocity and mean flow rate are considerably higher for H-B fluid model than those of the Casson fluid model.

(v) The estimates of the mean velocity and mean flow rate increase considerably with the increase of the body acceleration, and this behavior is reversed when the stenosis shape parameter increases.

Based on these results, one can note that there is substantial difference between the flow quantities of H-B fluid model and Casson fluid model, and thus it is expected that the use of H-B fluid model for blood flow in diseased artery may provide better results which may be useful to physicians in predicting the effects of body accelerations and different shapes and sizes of stenosis in the artery on the physiologically important flow quantities. Also, it is hoped that this study may provide some useful information to surgeons to take some crucial decisions regarding the treatment of patients, whether the cardiovascular disease can be treated with medicines or should the patient undergo a surgery. Hence, it is concluded that the present study can be treated as an improvement in the mathematical modeling of blood flow in narrow arteries with mild stenosis under the influence of periodic body accelerations.

\section{Nomenclature}

$\bar{r}$ : $\quad$ Radial distance

$r$ : $\quad$ Dimensionless radial distance

$\bar{z}$ : $\quad$ Axial distance

$z$ : $\quad$ Dimensionless axial distance

$n$ : Power law index

$\bar{p}: \quad$ Pressure

$p$ : Dimensionless pressure

$P: \quad$ Dimensionless pressure gradient

$\bar{Q}: \quad$ Flow rate

$Q: \quad$ Dimensionless flow rate

$\bar{R}_{0}$ : $\quad$ Radius of the normal artery

$\bar{R}(\bar{z})$ : Radius of the artery in the stenosed region

$R(z)$ : Dimensionless radius of the artery in the stenosed region

$F(\bar{t})$ : Body acceleration function

$a_{0}$ : Amplitude of the body acceleration

$\bar{R}_{P}$ : Plug core radius

$R_{P}$ : Dimensionless plug core radius

$\bar{u}_{H}$ : Axial velocity of Herschel-Bulkley fluid

$u_{H}$ : Dimensionless axial velocity of Herschel-Bulkley fluid

$\bar{u}_{C}:$ Axial velocity of Casson fluid

$u_{C}$ : Dimensionless axial velocity of Casson fluid

$\bar{A}_{0}$ : Steady component of the pressure gradient

$\bar{A}_{1}$ : Amplitude of the pulsatile component of the pressure gradient 
$\bar{L}$ : Length of the normal artery

$\bar{L}_{0}$ : Length of the stenosis

$m$ : Stenosis shape parameter

$L_{0}$ : Dimensionless length of the stenosis

$\bar{d}$ : Location of the stenosis

$d$ : Dimensionless location of the stenosis

$\bar{t}$ : Time

$t$ : Dimensionless time.

Greek Letters

$\Lambda:$ Dimensionless longitudinal impedance to flow

$\phi$ : Azimuthal angle

$\dot{\gamma}$ : Shear rate

$\bar{\tau}_{y}:$ Yield stress

$\theta$ : Dimensionless yield stress

$\bar{\tau}_{H}$ : Shear stress of the Herschel-Bulkley fluid

$\tau_{H}$ : Dimensionless shear stress of Herschel-Bulkley fluid

$\bar{\tau}_{C}$ : Shear stress for Casson fluid

$\tau_{C}$ : Dimensionless shear stress of Casson fluid

$\tau_{w}$ : Dimensionless wall shear stress

$\bar{\rho}_{H}$ : Density of Herschel-Bulkley fluid

$\bar{\rho}_{C}$ : Density of Casson fluid

$\bar{\mu}_{H}$ : Viscosity of Herschel-Bulkley fluid

$\bar{\mu}_{C}$ : Viscosity of the Casson fluid

$\alpha_{H}$ : Pulsatile Reynolds number of Herschel-Bulkley fluid

$\alpha_{C}$ : Pulsatile Reynolds number of Casson fluid

$\bar{\delta}$ : Depth of the stenosis

$\delta:$ Dimensionless depth of the stenosis

$\bar{\omega}$ : Angular frequency of the blood flow

$\phi$ : Lead angle.

Subscripts

$w$ : Wall shear stress (used for $\tau$ )

$H$ : Herschel-Bulkley fluid (used for $\bar{u}, u, \bar{\tau}, \tau$ )

$C$ : Newtonian fluid (used for $\bar{u}, u, \bar{\tau}, \tau$ ).

\section{Acknowledgments}

This research work was supported by the Research University Grant of Universiti Sains Malaysia, Malaysia (RU Grant ref. no. 1001/PMATHS/811177). The authors thank the reviewers for their valuable comments which helped to improve the technical quality of this research article. 


\section{References}

[1] S. Cavalcanti, "Hemodynamics of an artery with mild stenosis," Journal of Biomechanics, vol. 28, no. 4, pp. 387-399, 1995.

[2] M. E. Clark, J. M. Robertson, and L. C. Cheng, "Stenosis severity effects for unbalanced simplepulsatile bifurcation flow," Journal of Biomechanics, vol. 16, no. 11, pp. 895-906, 1983.

[3] D. Liepsch, M. Singh, and M. Lee, "Experimental analysis of the influence of stenotic geometry on steady flow," Biorheology, vol. 29, no. 4, pp. 419-431, 1992.

[4] G. T. Liu, X. J. Wang, B. Q. Ai, and L. G. Liu, "Numerical study of pulsating flow through a tapered artery with stenosis," Chinese Journal of Physics, vol. 42, no. 4 I, pp. 401-409, 2004.

[5] P. R. Johnston and D. Kilpatrick, "Mathematical modelling of flow through an irregular arterial stenosis," Journal of Biomechanics, vol. 24, no. 11, pp. 1069-1077, 1991.

[6] K. C. Ang and J. Mazumdar, "Mathematical modelling of triple arterial stenoses," Australasian Physical and Engineering Sciences in Medicine, vol. 18, no. 2, pp. 89-94, 1995.

[7] D. Kilpatrick, S. D. Webber, and J. P. Colle, "The vascular resistance of arterial stenoses in series," Angiology, vol. 41, no. 4, pp. 278-285, 1990.

[8] P. Chaturani and R. P. Samy, "Pulsatile flow of Casson's fluid through stenosed arteries with applications to blood flow," Biorheology, vol. 23, no. 5, pp. 499-511, 1986.

[9] D. S. Sankar and K. Hemalatha, "Pulsatile flow of Herschel-Bulkley fluid through stenosed arteries-A mathematical model," International Journal of Non-Linear Mechanics, vol. 41, no. 8, pp. 979-990, 2006.

[10] J. C. Misra and B. Pal, "A mathematical model for the study of the pulsatile flow of blood under an externally imposed body acceleration," Mathematical and Computer Modelling, vol. 29, no. 1, pp. 89-106, 1999.

[11] S. Chakravarty and P. K. Mandal, "Two-dimensional blood flow through tapered arteries under stenotic conditions," International Journal of Non-Linear Mechanics, vol. 35, no. 5, pp. 779-793, 2000.

[12] V. K. Sud and G. S. Sekhon, "Arterial flow under periodic body acceleration," Bulletin of Mathematical Biology, vol. 47, no. 1, pp. 35-52, 1985.

[13] R. Usha and K. Prema, "Pulsatile flow of particle-fluid suspension model of blood under periodic body acceleration," Zeitschrift fur Angewandte Mathematik und Physik, vol. 50, no. 2, pp. 175-192, 1999.

[14] P. K. Mandal, S. Chakravarty, A. Mandal, and N. Amin, "Effect of body acceleration on unsteady pulsatile flow of non-newtonian fluid through a stenosed artery," Applied Mathematics and Computation, vol. 189, no. 1, pp. 766-779, 2007.

[15] N. Mustapha, S. Chakravarty, P. K. Mandal, and N. Amin, “Unsteady response of blood flow through a couple of irregular arterial constrictions to body acceleration," Journal of Mechanics in Medicine and Biology, vol. 8, no. 3, pp. 395-420, 2008.

[16] M. El-Shahed, "Pulsatile flow of blood through a stenosed porous medium under periodic body acceleration," Applied Mathematics and Computation, vol. 138, no. 2-3, pp. 479-488, 2003.

[17] V. P. Rathod, S. Tanveer, I. S. Rani, and G. G. Rajput, "Pulsatile flow of blood under periodic body acceleration and magnetic field through an exponentially diverging vessel," Proceedings National Conference Advanced, Fluid Dynamics, pp. 106-117, 2004.

[18] S. Chakravarty and A. K. Sannigrahi, "An analytical estimate of the flow-field in a porous stenotic artery subject to body acceleration," International Journal of Engineering Science, vol. 36, no. 10, pp. 1083-1102, 1998.

[19] P. Chaturani and V. Palanisamy, "Pulsatile flow of blood with periodic body acceleration," International Journal of Engineering Science, vol. 29, no. 1, pp. 113-121, 1991.

[20] S. N. Majhi and V. R. Nair, "Pulsatile flow of third grade fluids under body acceleration-Modelling blood flow," International Journal of Engineering Science, vol. 32, no. 5, pp. 839-846, 1994.

[21] C. Tu and M. Deville, "Pulsatile flow of Non-Newtonian fluids through arterial stenoses," Journal of Biomechanics, vol. 29, no. 7, pp. 899-908, 1996.

[22] D. S. Sankar and K. Hemalatha, "Pulsatile flow of Herschel-Bulkley fluid through catheterized arteries-a mathematical model," Applied Mathematical Modelling, vol. 31, no. 8, pp. 1497-1517, 2007.

[23] P. Chaturani and A. S. A. Wassf Isaac, "Blood flow with body acceleration forces," International Journal of Engineering Science, vol. 33, no. 12, pp. 1807-1820, 1995.

[24] G. Sarojamma and P. Nagarani, "Pulsatile flow of Casson fluid in a homogenous porous medium subject to external acceleration," International Journal of Non-Linear Differential Equations, vol. 7, pp. 50-64, 2002. 
[25] P. Chaturani and V. Palanisamy, "Casson fluid model for pulsatile flow of blood under periodic body acceleration," Biorheology, vol. 27, no. 5, pp. 619-630, 1990.

[26] P. Chaturani and V. Palanisamy, "Pulsatile flow of power-law fluid model for blood flow under periodic body acceleration," Biorheology, vol. 27, no. 5, pp. 747-758, 1990.

[27] S. Chakravarty and P. K. Mandal, "A nonlinear two-dimensional model of blood flow in an overlapping arterial stenosis subjected to body acceleration," Mathematical and Computer Modelling, vol. 24, no. 1, pp. 43-58, 1996.

[28] Y. C. Fung, Biomechanics: Mechanical Properties of Living Tissues, Springer, Berlin, Germany, 1981.

[29] J. N. Kapur, Mathematical Models in Biology and Medicine, Affiliated East-West Press Pvt. Ltd., New Delhi, India, 1992.

[30] N. Iida, "Influence of plasma layer on steady blood flow in micro vessels," Japanese Journal of Applied Physics, vol. 17, pp. 203-214, 1978.

[31] D. S. Sankar and K. Hemalatha, "A non-Newtonian fluid flow model for blood flow through a catheterized artery-Steady flow," Applied Mathematical Modelling, vol. 31, no. 9, pp. 1847-1864, 2007.

[32] D. S. Sankar and A. I. M. Ismail, "Effect of periodic body acceleration in blood flow through stenosed arteries-a theoretical model," International Journal of Nonlinear Sciences and Numerical Simulation, vol. 11, no. 4, pp. 243-257, 2010.

[33] P. Nagarani and G. Sarojamma, "Effect of body acceleration on pulsatile flow of casson fluid through a mild stenosed artery," Korea Australia Rheology Journal, vol. 20, no. 4, pp. 189-196, 2008.

[34] D. S. Sankar and U. Lee, "Mathematical modeling of pulsatile flow of non-Newtonian fluid in stenosed arteries," Communications in Nonlinear Science and Numerical Simulation, vol. 14, no. 7, pp. 2971-2981, 2009.

[35] S. U. Siddiqui, N. K. Verma, S. Mishra, and R. S. Gupta, "Mathematical modelling of pulsatile flow of Casson's fluid in arterial stenosis," Applied Mathematics and Computation, vol. 210, no. 1, pp. 1-10, 2009. 


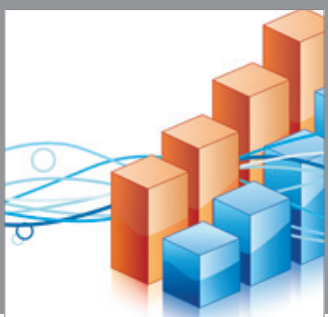

Advances in

Operations Research

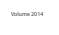

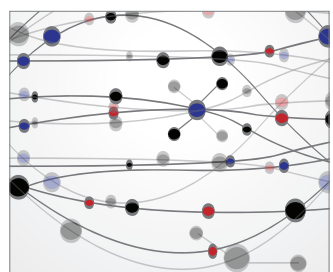

\section{The Scientific} World Journal
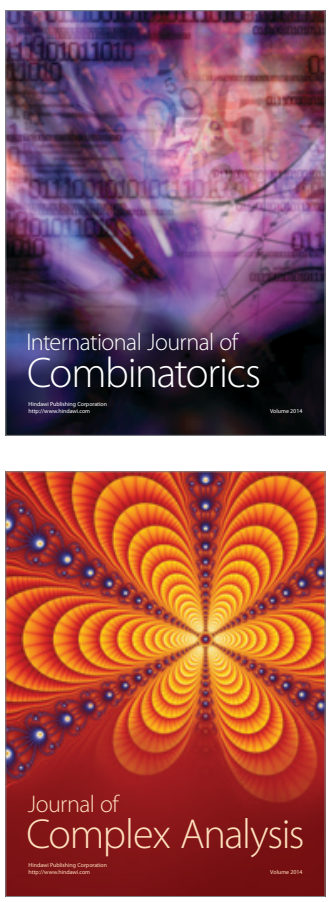

International Journal of

Mathematics and

Mathematical

Sciences
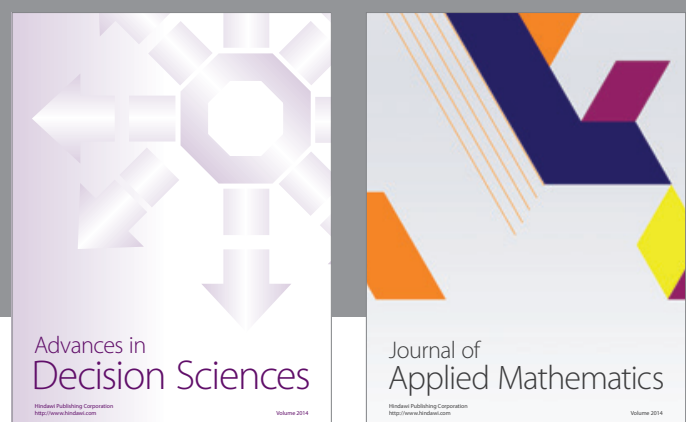

Journal of

Applied Mathematics
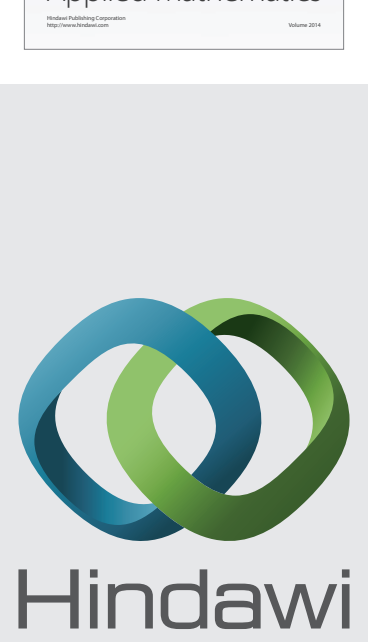

Submit your manuscripts at http://www.hindawi.com
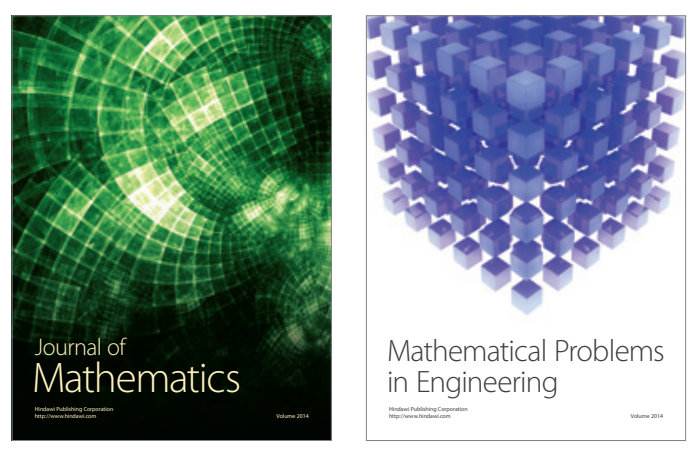

Mathematical Problems in Engineering
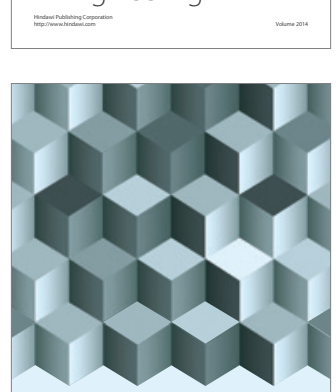

Journal of

Function Spaces
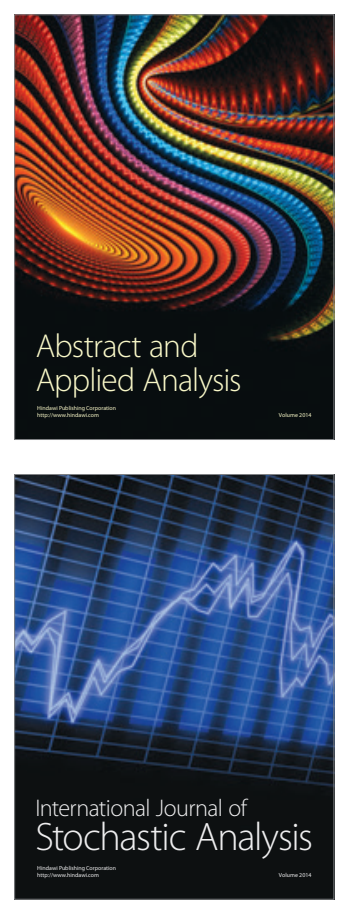

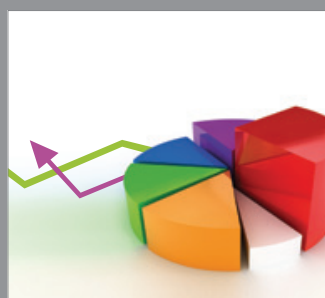

ournal of

Probability and Statistics

Promensencen
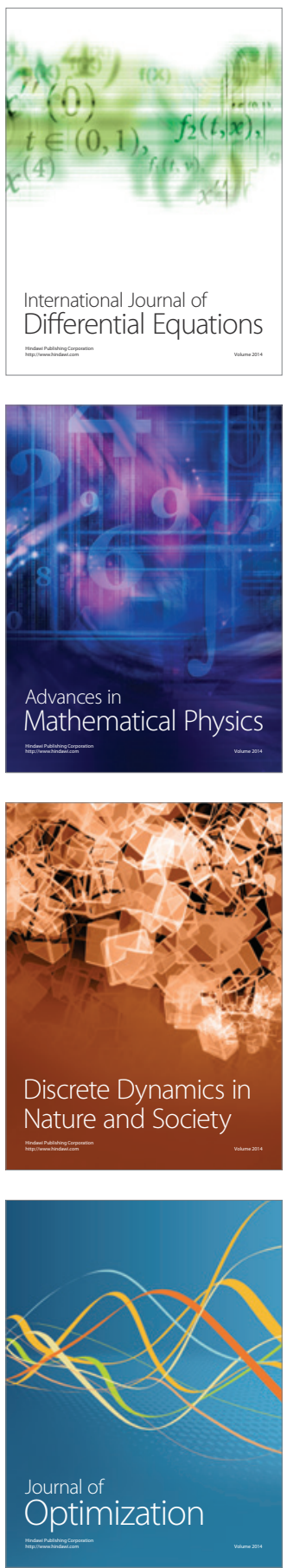